\title{
Tendência de busca sobre nutrição durante a pandemia de COVID-19 no Brasil:
}

\section{resultados do Google trends}

\author{
Search trend on nutrition during the COVID-19 pandemic in Brazil: Google trends results \\ Tendencia de búsqueda en nutrition durante la pandemia del COVID-19 en Brasil: resultados de
} Google trends

Recebido: 18/09/2021 | Revisado: 25/09/2021 | Aceito: 27/09/2021 | Publicado: 28/09/2021

Vinícius Eponina dos Santos ORCID: https://orcid.org/0000-0002-8111-6464 Universidade Federal de Sergipe, Brasil E-mail: viniciuseponina@gmail.com Liliane Viana Pires

ORCID: https://orcid.org/0000-0003-1710-0836 Universidade Federal de Sergipe, Brasil E-mail: lvianapires@academico.ufs.br

Vivianne de Sousa Rocha

ORCID: https://orcid.org/0000-0003-3016-3268 Universidade Federal de Sergipe, Brasil

E-mail: vrocha@academico.ufs.br

\begin{abstract}
Resumo
Introdução: O Google Trends (GT) é uma ferramenta muito utilizada para pesquisa de tendência de busca de informações. Entretanto, poucos estudos investigaram as tendências de busca associando temas de Nutrição ao COVID-19. Objetivo: Investigar os temas envolvendo Nutrição no período da pandemia da COVID-19 no Brasil. Métodos: Inicialmente, foram pesquisados os temas de Nutrição mais buscados na internet, baseando-se em notícias, matérias e artigos. Em seguida, esses termos foram aplicados no GT, considerando o período de 01 de março de 2020 a 20 de junho de 2021, e a geolocalização. Os dados do GT foram avaliados pelos picos de interesse ao longo do período analisado. Resultados: Os termos mais citados no Google foram: segurança alimentar, vitamina D, fitoterapia e alimentação saudável. Ao serem aplicados no GT, observou-se que os temas segurança alimentar e fitoterapia permaneceram com elevado volume de busca desde o início da pandemia em 2020 até meados de 2021. Já o termo vitamina D e alimentação saudável apresentaram picos de interesse em períodos variados. Quanto a geolocalização das buscas, o estado da Paraíba foi o que apresentou o maior interesse entre os termos analisados. Conclusão: O GT tem se mostrado uma ferramenta para avaliar o comportamento social. Os assuntos segurança alimentar, fitoterapia e alimentação saudável apresentaram elevada tendência de busca durante a pandemia da COVID-19, enquanto, o termo vitamina $\mathrm{D}$ teve um pico de interesse, apenas no início da pandemia.
\end{abstract}

Palavras-chave: Internet; Nutrição, COVID-19.

\begin{abstract}
Introduction: Google Trends (GT) is a widely used tool for trend research. However, few studies have investigated search trends associating Nutrition themes with COVID-19. Objective: To investigate the themes involving Nutrition in the period of the COVID-19 pandemic. Methods: Initially, the most searched Nutrition topics on the internet were researched, based on news, publications, and articles. After, the terms were applied to the GT, considering the period from March 1, 2020, to June 20, 2021, and geolocation. The GT data were evaluated by the peaks of interest to the analyzed period. Results: The most cited terms on Google were food safety, vitamin D, herbal medicine, and healthy eating. When applied in the GT, it was observed that the themes of food safety and herbal medicine remained with a high volume of search from the beginning of the pandemic in 2020 to 2021. The terms vitamin D and healthy eating showed peaks of interest at different periods. As for the geolocation, the state of Paraíba was the state that showed the greatest interest among the terms analyzed. Conclusion: The GT has been shown to be a tool to assess social behavior. The subject's food safety, herbal medicine, and healthy eating showed a high search trend during the COVID-19 pandemic, while the term vitamin D had a peak of interest only at the beginning of the pandemic.
\end{abstract}

Keywords: Internet; Nutrition, COVID-19.

\section{Resumen}

Introducción: Google Trends (GT) es una herramienta ampliamente utilizada para la búsqueda de información de tendencias. Sin embargo, pocos estudios han investigado las tendencias de búsqueda que asocian temas de nutrición 
con COVID-19. Objetivo: Investigar los temas relacionados con la Nutrición en el período de la pandemia de COVID-19 en Brasil. Métodos: Inicialmente, se investigaron los temas de nutrición más buscados en Internet, a partir de noticias, artículos y artículos. Luego, estos términos se aplicaron en el GT, considerando el período del 1 de marzo de 2020 al 20 de junio de 2021 y la geolocalización. Los datos de TG fueron evaluados por los picos de interés a lo largo del período analizado. Resultados: Los términos más citados en Google fueron: seguridad alimentaria, vitamina D, hierbas medicinales y alimentación saludable. Cuando se aplicó en el GT, se observó que los temas seguridad alimentaria y fitoterapia se mantuvieron con un alto volumen de búsqueda desde el inicio de la pandemia en 2020 hasta mediados de 2021. El término vitamina D y alimentación saludable, por otro lado, mostró picos de interés en diferentes períodos. En cuanto a la geolocalización de las búsquedas, el estado de Paraíba fue el que mostró mayor interés entre los términos analizados. Conclusión: Se ha demostrado que el GT es una herramienta para evaluar el comportamiento social. Los sujetos seguridad alimentaria, medicina herbal y alimentación saludable mostraron una alta tendencia de búsqueda durante la pandemia COVID-19, mientras que el término vitamina D tuvo un pico de interés solo al comienzo de la pandemia.

Palabras clave: Internet; Nutrición, COVID-19.

\section{Introdução}

A internet sempre foi utilizada para a busca de informação em diversos assuntos, e mais recentemente também tem crescido o seu uso como geradora de dados para pesquisas de tendência sobre temas de interesse da população. Quando essa busca por informações é sobre saúde, denomina-se de infodemiologia, sendo definida como "a ciência da distribuição e determinantes da informação em um meio eletrônico, especificamente a internet, ou em uma população, com o objetivo final de informar o público sobre saúde e políticas públicas” (Mavragani \& Ochoa, 2019).

O Google Trends (GT), uma interface do Google, vem se destacado dentre as ferramentas mais utilizadas para pesquisa de tendência, por ter livre acesso e ser de fácil manuseio, baseado em consultas reais dos usuários, geolocalizados, com categorias temáticas (notícias, saúde, ciência, entre outras) e com delimitações temporais. Quando o termo, a região, o período e a categoria são definidos, os resultados geram um gráfico das variações de todos os termos examinados ao longo do período selecionado (Mavragani \& Ochoa, 2019; Passos, Vasconcellos-Silva \& Santos, 2020), podendo verificar a tendência de busca por informações relativas ao tema de acordo com as categorias delimitadas.

Durante a pandemia do coronavírus-19 (COVID-19) a busca por informações sobre formas de prevenção e manutenção da saúde tiverem grande repercussão na internet (Passos et al., 2020). Dentre os aspectos de saúde, diversos temas sobre Nutrição relacionados à pandemia de COVID-19 surgiram nas mídias, como a alimentação saudável e a insegurança alimentar. No entanto, até o momento, poucos estudos investigaram as tendências de busca associando temas relacionados à Nutrição e a COVID-19, principalmente no Brasil, país que apresentou a soma de fatores agravantes, alta taxa de transmissão da doença e baixa adesão às medidas de isolamento social (Rahimi Pordanjan et al., 2021).

Dessa forma, este artigo buscou investigar os temas envolvendo Nutrição durante o período da pandemia da COVID19, com os seguintes objetivos: (1) selecionar os tópicos mais citados sobre Nutrição no buscador Google no Brasil, (2) analisar no GT o volume de dados de interesse dos tópicos selecionados e (3) explorar a distribuição geográfica de pesquisa desses tópicos no Brasil.

\section{Metodologia}

\subsection{Coleta de termos}

Inicialmente, foram selecionados os principais temas atualmente relacionados à Nutrição e a COVID-19 no buscador Google. Para tanto, considerou-se as notícias publicadas em jornais eletrônicos e notícias de Universidades como Universidade de São Paulo (USP), Universidade Federal de Pernambuco (UFPE), Universidade Federal Fluminense (UFF), assim como artigos publicados envolvendo a alimentação/nutrição com o contexto atual. 


\subsection{Dados no Google Trends}

Após a seleção dos termos com maior repercussão avaliada no buscador Google, foi estimado o Volume Relativo de Busca (VRB) pelo GT, que é entendido como o nível de interesse a respeito do tema (Kamiński, Skonieczna-Żydecka, Nowak \& Stachowska, 2020). O VRB é um índice de volume de pesquisa ajustado ao número de usuários do Google em uma determinada área geográfica, variando de 0 a 100 no pico de popularidade. Os resultados foram apresentados nesse intervalo, sendo que o valor de 100 indica $100 \%$ de popularidade em determinado período e local e o 0 desinteresse total ( $0 \%$ ) (Nuti et al., 2014).

Além disso, o GT pode qualificar as frases analisadas como "termo de pesquisa" ou "tópico". Os termos de pesquisa são palavras digitadas literalmente, enquanto os tópicos podem ser propostos pelo GT quando a ferramenta reconhece frases relacionadas a consultas populares. A categoria permite uma melhor especificidade do que se deseja observar sobre um determinado termo entre países e estados (Arshad et al., 2020).

Os termos pesquisados neste estudo foram aplicados no GT, no período de 69 semanas (de 01 de março de 2020 a 20 de junho de 2021), selecionando a região (Brasil) e a categoria de assunto (Saúde). Na coleta de dados foi realizada uma busca "limpa" após limpeza dos dados de navegação e cookies que poderiam influenciar o histórico de buscas.

\subsection{Análise dos dados}

Os dados fornecidos pelo GT foram importados como arquivos CSV (Comma Separated Values) para planilhas eletrônicas (MS Excel®) e foram avaliados os picos de interesse ao longo do período selecionado, assim como as linhas de tendência. As curvas dos temas foram tabuladas em quatro séries independentes, tendo em vista que o GT produz resultados comparando curvas de acessos ao pico de maior popularidade, o que poderia subestimar as estimativas dos temas menos populares. Os resultados foram apresentados em infográficos com os principais picos de interesse dos termos em questão.

\section{Resultados}

Foram selecionados os termos com maior repercussão: (1) segurança alimentar, (2) vitamina D, (3) fitoterapia e (4) alimentação saudável. A partir do GT foi possível notar que o termo segurança alimentar permaneceu com alto volume de busca, desde que a OMS decretou a pandemia de COVID-19 no mundo em março de 2020 até meados de junho de 2021, com o maior pico de interesse em outubro de 2020 (Gráfico 1). A Vitamina D apresentou seus principais picos no início da pandemia de COVID-19, decrescendo gradativamente com o passar dos meses, não passando de 45 no VRB até o fim do período avaliado (Gráfico 2).

Ao contrário do tema vitamina $\mathrm{D}$, o assunto fitoterapia permaneceu com elevada tendência de busca no decorrer dos meses avaliados, principalmente entre os meses de março a setembro de 2020, no qual apresentou o seu principal pico (100 no

VRB) (Gráfico 3). Os picos de interesse sobre alimentação saudável aconteceram a partir do mês de junho de 2020, permanecendo relativamente alto nos meses seguintes (Gráfico 4).

Em relação à distribuição geográfica das tendências de buscas no Brasil, a região nordeste apresentou mais buscas para o tema de alimentação saudável, em destaque o Estado da Paraíba, que apresentou maior interesse de busca pelos temas, exceto para o termo fitoterapia. A vitamina $\mathrm{D}$ foi o assunto mais pesquisado nas regiões sul e sudeste. Em relação às demais regiões, houve grande variação entre os interesses de busca para os temas (Gráficos 5, 6, 7 e 8). A Tabela 1 traz o checklist baseado em Nuti et al. (2014) para pesquisas realizadas no GT. 
Gráfico 1 - Picos de interesse do termo segurança alimentar.

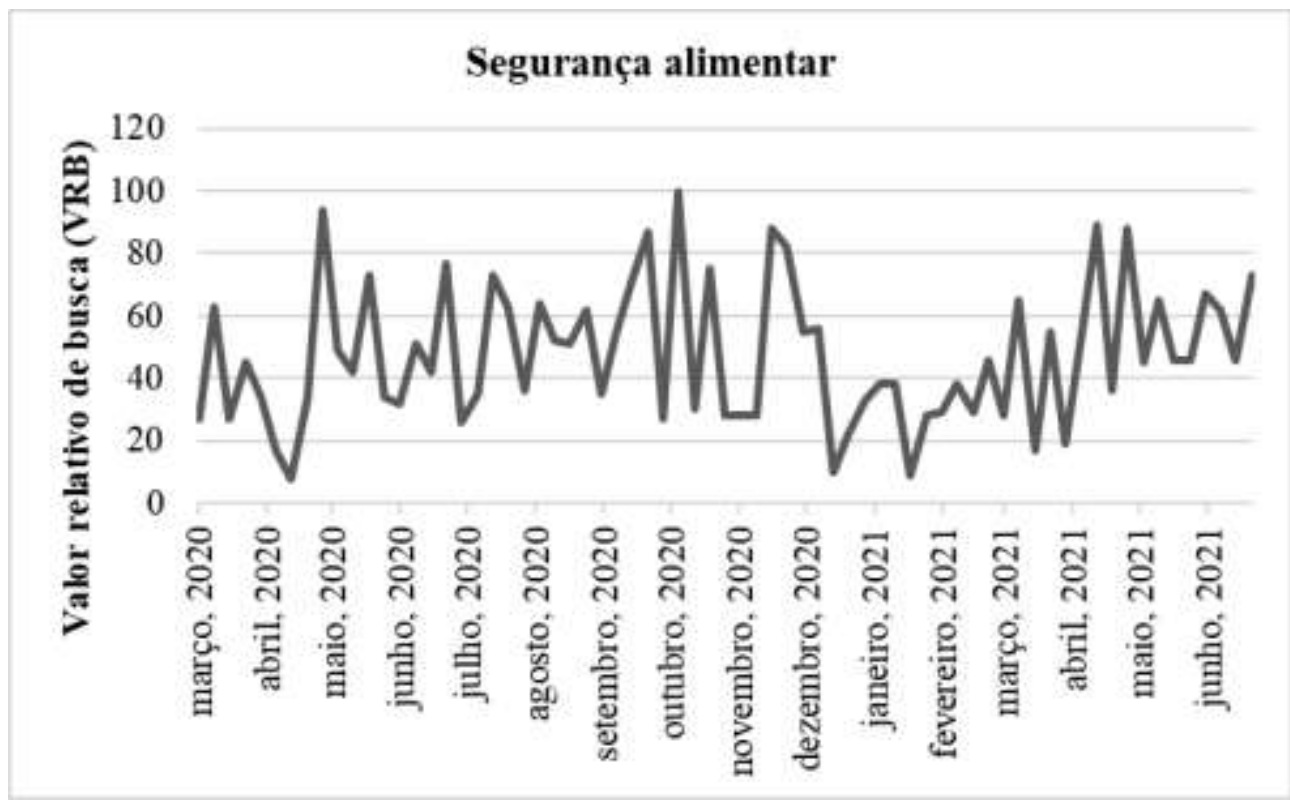

Fonte: Autores.

Gráfico 2 - Picos de interesse do termo vitamina D.

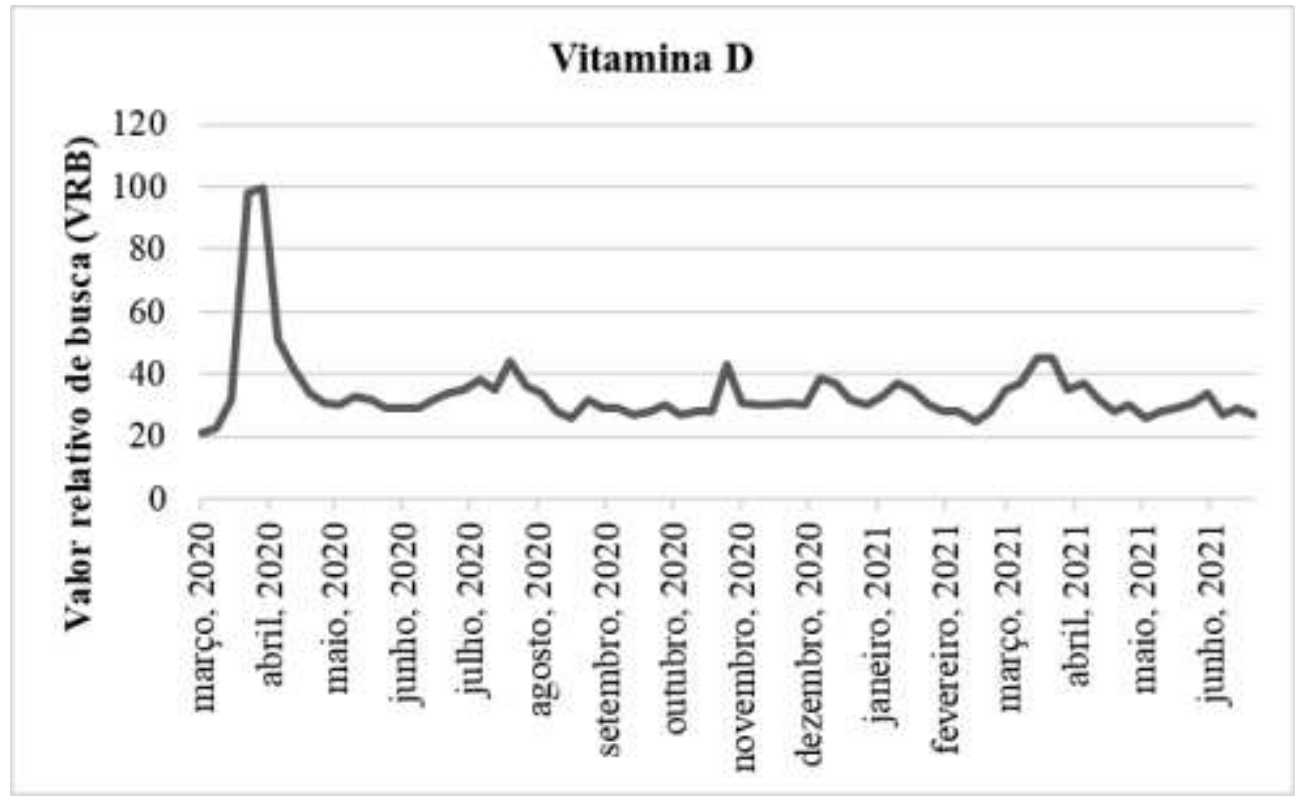

Fonte: Autores. 
Gráfico 3 - Picos de interesse do termo Fitoterapia.

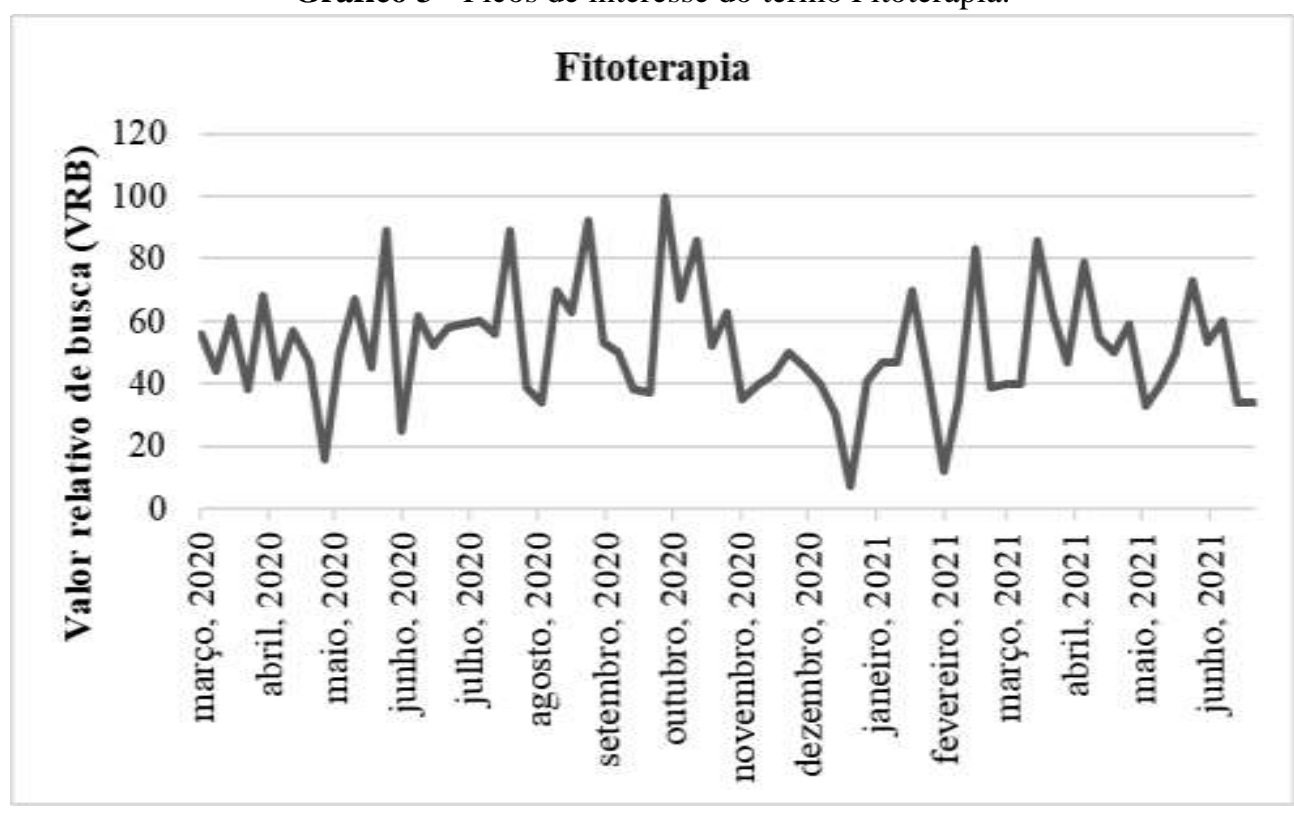

Fonte: Autores.

Gráfico 4 - Picos de interesse do termo alimentação saudável.

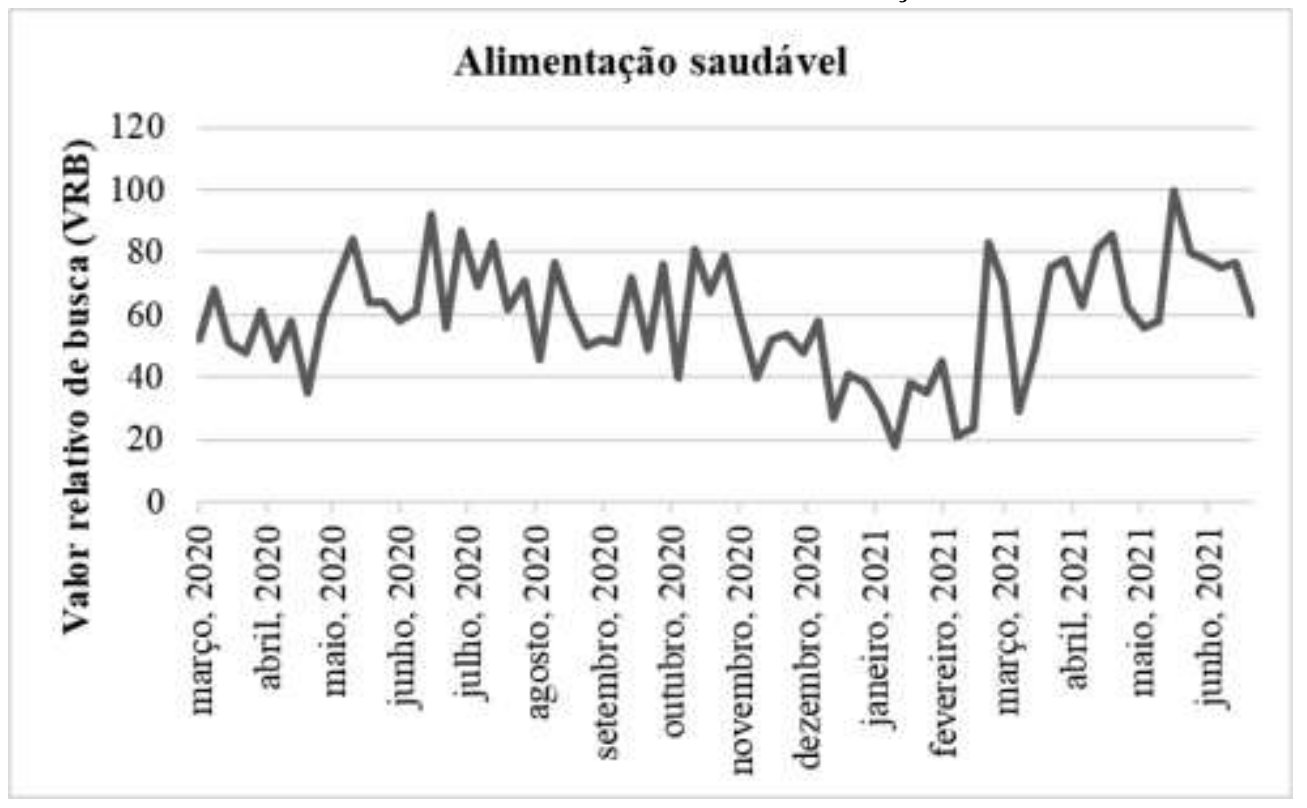

Fonte: Autores. 
Gráfico 5 - Distribuição geográfica do termo "Segurança alimentar" durante o período da pandemia de COVID-19 (março de 2020 a junho de 2021) no Brasil. Legenda: VRB (Valor Relativo de Busca).

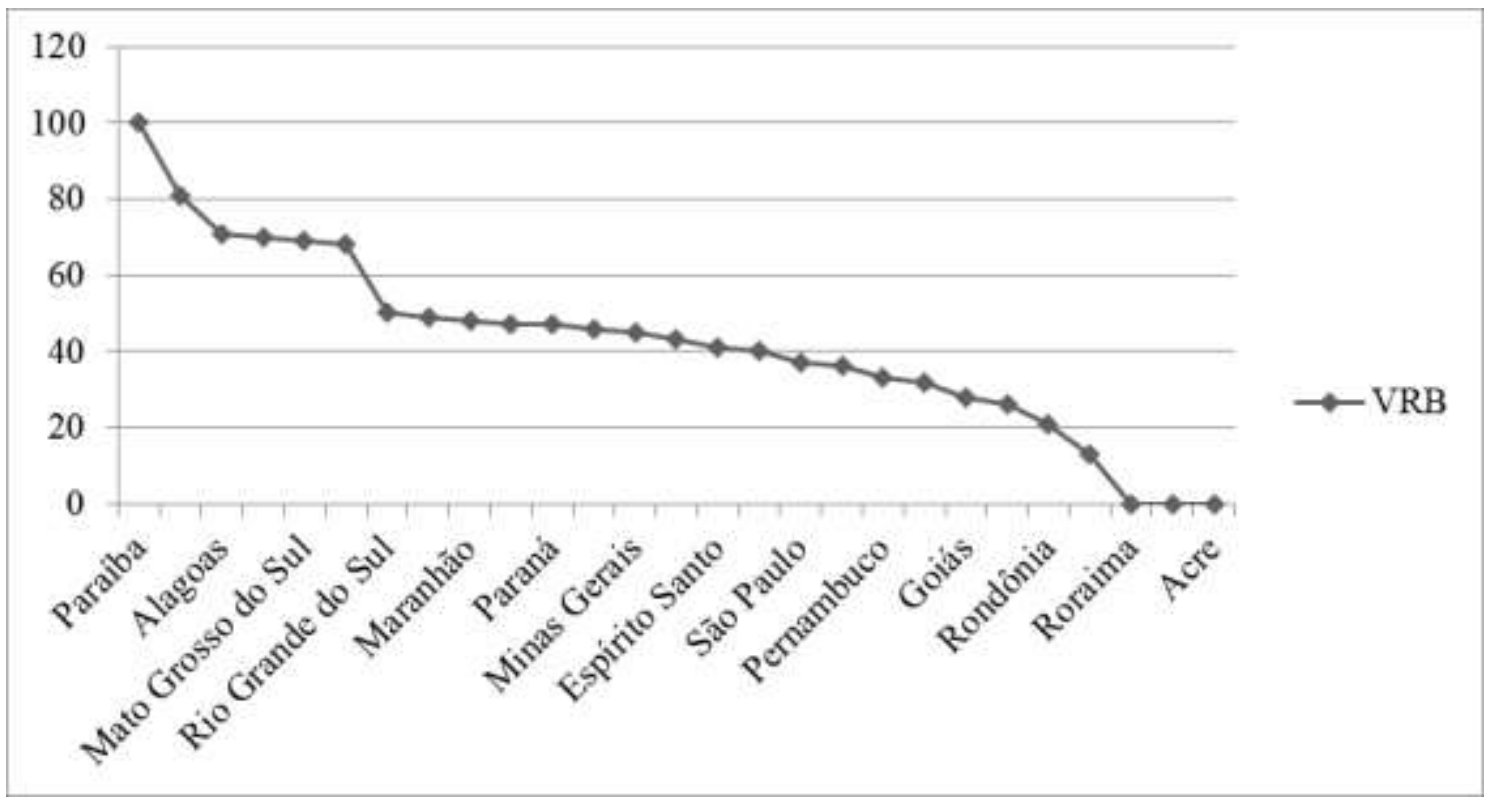

Fonte: Autores.

Gráfico 6 - Distribuição geográfica do termo "Vitamina D” durante o período da pandemia de COVID-19 (março de 2020 a junho de 2021) no Brasil. Legenda: VRB (Valor Relativo de Busca).

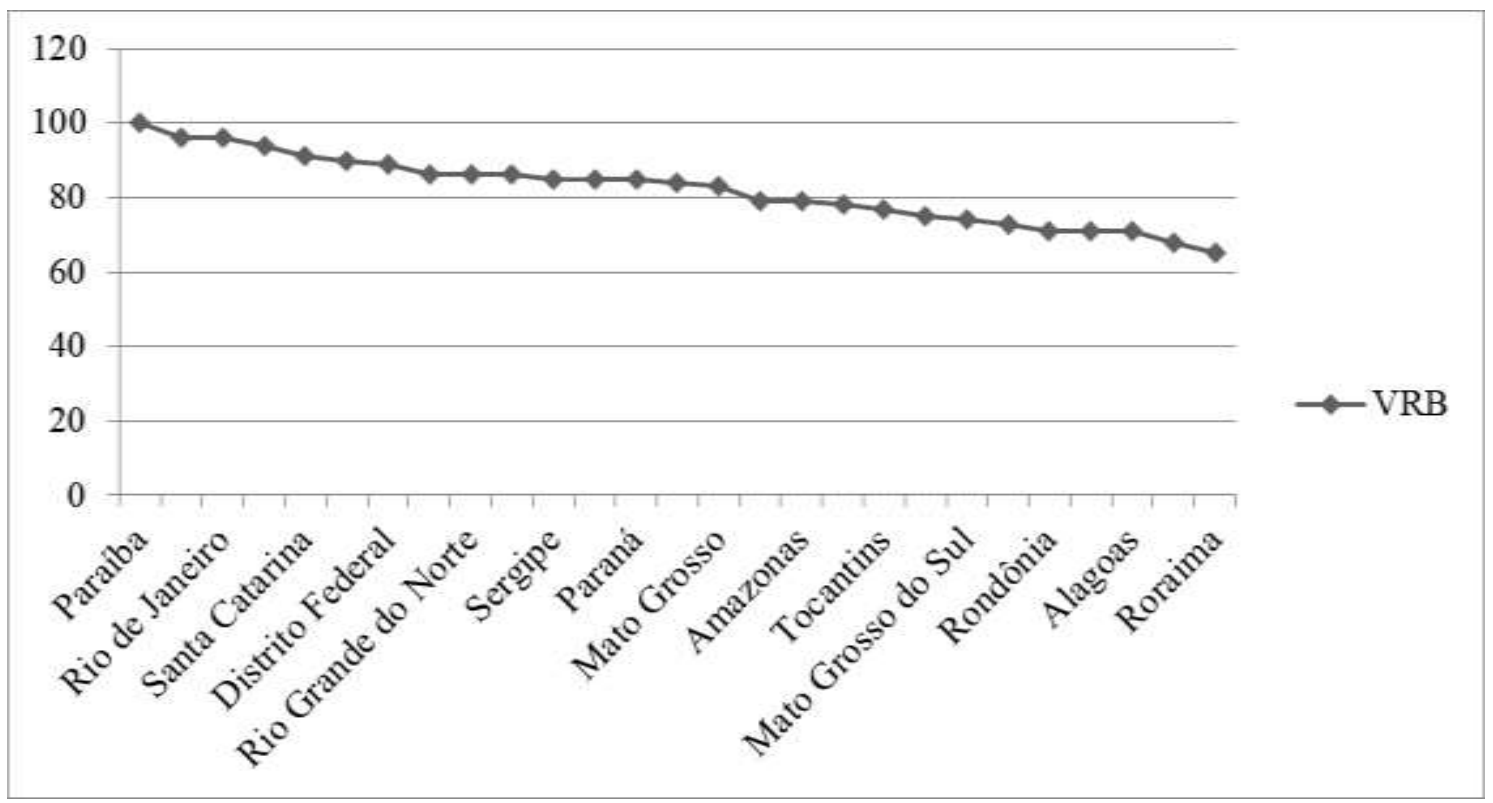

Fonte: Autores. 
Gráfico 7 - Distribuição geográfica do termo "Fitoterapia" durante o período da pandemia de COVID-19 (março de 2020 a junho de 2021) no Brasil. Legenda: VRB (Valor Relativo de Busca).

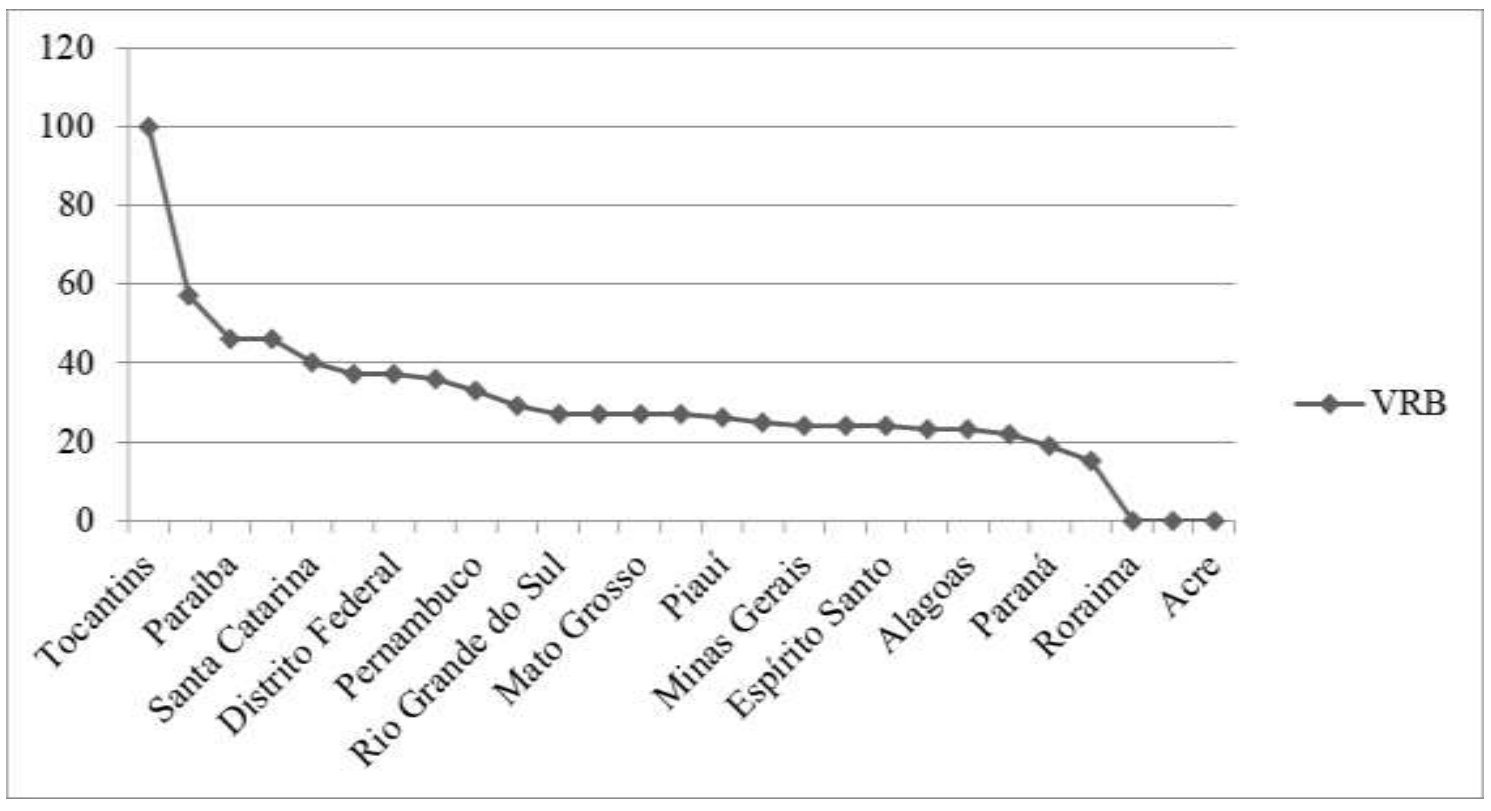

Fonte: Autores.

Gráfico 8 - Distribuição geográfica do termo "Alimentação saudável" durante o período da pandemia de COVID-19 (março de 2020 a junho de 2021) no Brasil. Legenda: VRB (Valor Relativo de Busca).

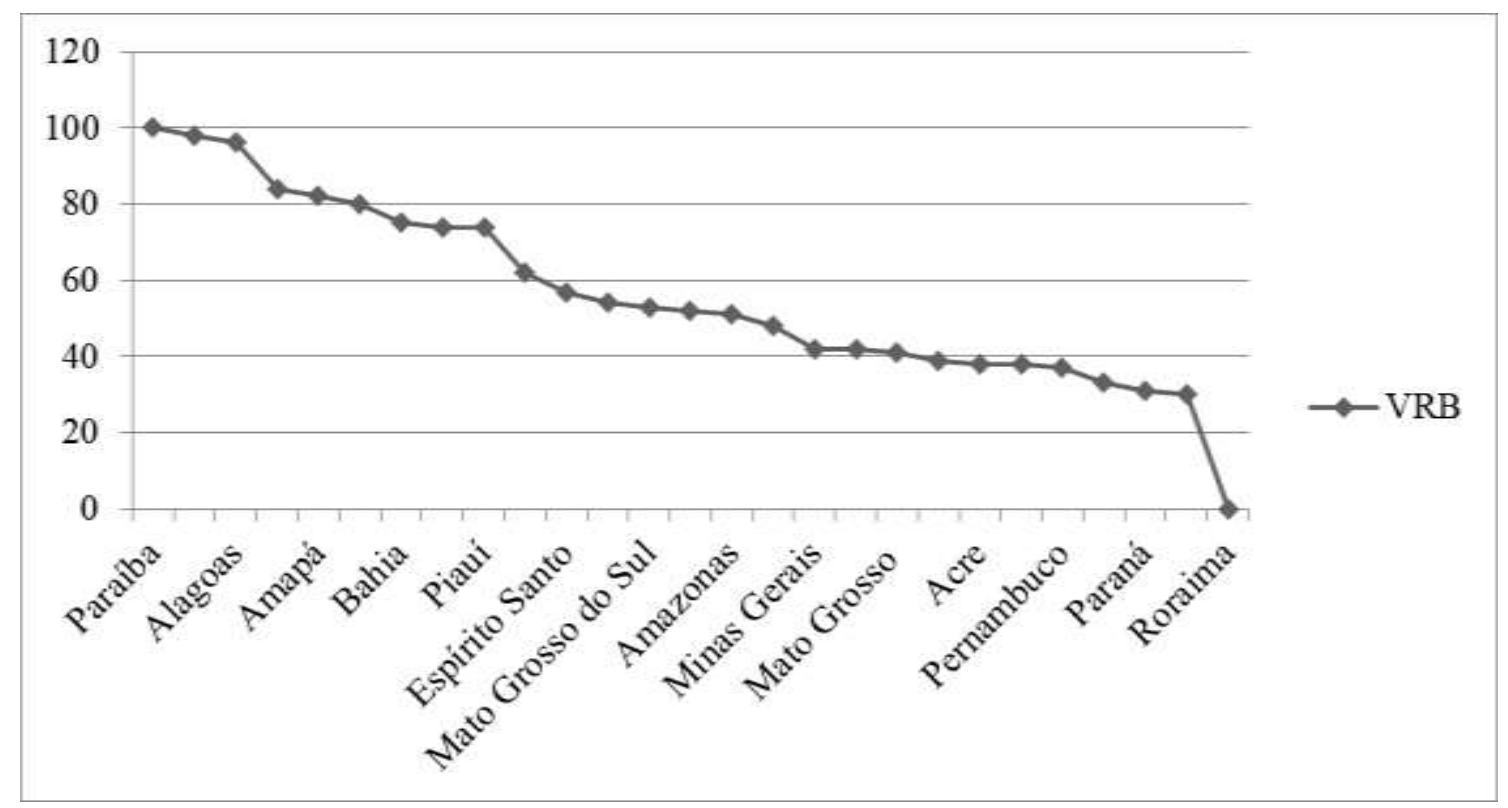

Fonte: Autores. 
Tabela 1 - Checklist baseado em Nuti et al. (2014) para pesquisas realizadas no GT.

\begin{tabular}{|c|c|}
\hline $\begin{array}{c}\text { Seção/ tópico } \\
\text { Variáveis pesquisadas }\end{array}$ & Checklist item \\
\hline $\begin{array}{l}\text { Data do acesso } \\
\text { Período de tempo } \\
\text { Categoria pesquisada } \\
\text { Região }\end{array}$ & $\begin{array}{c}\text { 28/06/2021 } \\
\text { De } 01 \text { de março de } 2020 \text { a } 20 \text { de junho de } 2021 \\
\text { Saúde } \\
\text { Brasil }\end{array}$ \\
\hline Dados coletados & $\begin{array}{l}\text { Tópicos “segurança alimentar”, "vitamina D”, } \\
\text { "Fitoterapia", "alimentação saudável”. }\end{array}$ \\
\hline Justificativa para a estratégia de busca & $\begin{array}{c}\text { O tópico "Segurança alimentar" refere-se ao tema } \\
\text { Segurança Alimentar e Nutricional (SAN). Foi } \\
\text { usado para facilitar a busca. }\end{array}$ \\
\hline Para pesquisa inicial & $\begin{array}{l}\text { Usou o site do Google para analisar os principais } \\
\text { tópicos da Nutrição que tiveram grande } \\
\text { repercussão na mídia e posteriormente aplicou-se } \\
\text { no GT para verificar a tendência de busca. }\end{array}$ \\
\hline \multirow[t]{2}{*}{ Para configuração escolhida } & \\
\hline & $\begin{array}{l}\text { Utilizou-se a categoria "Saúde" e a região } \\
\text { "Brasill", a fim de especificar os dados que se } \\
\text { pretenderam analisar, assim como para evitar a } \\
\text { aparição de dados que não fazem parte do } \\
\text { objetivo do trabalho. }\end{array}$ \\
\hline
\end{tabular}

Fonte: Autores.

\section{Discussão}

De acordo com os resultados apresentados, observa-se que os temas mais pesquisados pelos brasileiros foram: segurança alimentar, vitamina D, fitoterapia e alimentação saudável. Além disso, nota-se que houve picos diferentes para cada tema, sendo que apenas os termos segurança alimentar e alimentação saudável permanecem com elevados picos até o final do período analisado.

A pandemia por COVID-19 representa um dos maiores desafios da saúde pública deste século (Angelini \& Zago, 2021). No Brasil, a pandemia amplificou as desigualdades sociais já existentes, comprometendo ainda mais a garantia do Direito Humano à Alimentação Adequada (DHAA) e a concretização da Segurança Alimentar e Nutricional (SAN), especialmente entre os mais vulneráveis (Santos, Oliveira, Pereira \& Santos, 2021). Fato que justifica o interesse pela busca do tema em razão do cenário atual gerado pela pandemia de COVID-19, que aumenta a insegurança alimentar e nutricional devido ao agravamento da situação de pobreza, da fome e da falta de acesso aos alimentos, bem como pelo excesso do consumo de alimentos ultraprocessados, gerando uma preocupação tanto pela população em geral quanto pela comunidade científica (Gurgel, Santos, Alves, Araujo \& Leal, 2020). Tal situação é contrária ao fundamento da SAN, que destaca o direito de todos ao acesso regular e permanente a alimentos de qualidade, em quantidade suficiente, sem comprometer o acesso a outras necessidades essenciais (Brasil, 2006).

Outro tema em evidência na pandemia foi a alimentação saudável, que é entendida como aquela que contempla todas as necessidades biológicas, sociais e emocionais dos indivíduos, ou seja, deve ser variada, equilibrada, suficiente, acessível, 
colorida e segura, sendo uma fonte de prazer e identidade cultural e familiar, podendo reduzir o risco do aparecimento de doenças, sendo essencial para promover e manter a saúde (OPAS, 2019; Brasil, 2020). Nesse sentido, a alimentação saudável influencia substancialmente de maneira positiva a saúde do indivíduo, sendo a boa nutrição um fator significativo na determinação do estado de saúde e longevidade neste período de pandemia (OPAS, 2019; Sousa et al., 2021). Dessa forma, a reflexão acerca da importância de uma alimentação saudável, tendo em vista a manutenção da saúde, o fortalecimento do sistema imunológico, além da necessidade de aplicação de medidas de higiene para evitar contaminações é um dos motivos da elevada busca pelo tema (Brasil, 2020).

A fitoterapia também esteve entre os termos mais pesquisados entre os meses de 2020. Esse termo entrou no contexto da nutrição devido os fitoterápicos serem preparados exclusivamente com plantas ou partes de plantas medicinais, que possuem propriedades reconhecidas de cura, prevenção, diagnóstico ou tratamento sintomático de doenças (Brasil, 2004; Braga \& Silva, 2021). Dentre as propriedades biológicas dos fitoterápicos estão as de estimular as reações do sistema imune, entre elas, ações imunomoduladoras, conferindo um aumento da resposta imunológica do indivíduo (Valeriano, Savani \& Silva, 2019). Sendo assim, o interesse da população a respeito do tema pode ser devido às propriedades dos fitoterápicos e a sua possível relação com o COVID-19. No entanto, mais estudos são necessários para verificar essa associação.

Diferentemente dos temas citados acima, o termo vitamina D esteve em alta apenas no início da pandemia, apresentando seu pico no mês de março. A vitamina D é uma vitamina lipossolúvel essencial para o metabolismo de ossos e minerais (Manson, Brannon, Rosen \& Taylor, 2016), mas a sua deficiência está relacionada na patogênese de diversas doenças (Jung et al., 2021). Foi notória a discussão entre a comunidade científica a respeito da relação entre a vitamina $\mathrm{D}$ e o coronavírus, já que além de ser imprescindível na homeostase do metabolismo ósseo, a vitamina D tem participação no controle de processos inflamatórios e imunológicos (Carpagnano et al., 2021). Entretanto, até o momento não há estudos que comprovem a prevenção e/ou tratamento da COVID-19 por meio da suplementação da vitamina D (Lima, 2021).

A variação no pico de interesse em alguns temas pode ser acompanhada pelos períodos críticos, como o início da pandemia quando as pessoas não tinham muitas informações a respeito das formas de manutenção da saúde nessa situação pandêmica e em períodos de maior transmissão e morte pela COVID-19, como entre os meses de maio a agosto de 2020 (primeira "onda") e entre fevereiro e junho de 2021 (https://bigdata-covid19.icict.fiocruz.br).

No Brasil, o estado com maior volume de busca foi o da Paraíba, no qual se manteve em primeiro lugar em todos os termos, exceto o da fitoterapia. A alta procura pelos temas citados pode ser devido ao cenário gerado pela pandemia de COVID-19, sendo reconhecida como um desafio enorme para os países, principalmente para o Brasil.

\section{Conclusão}

Conclui-se que os temas mais buscados no Brasil relacionados à Nutrição na pandemia de COVID-19 foram sobre segurança alimentar, vitamina D, fitoterapia e alimentação saudável com picos de buscas considerados elevados em determinados períodos do intervalo de tempo avaliado. A longa duração da pandemia pode ter contribuído para as variações de buscas nos temas. Ressalta-se que o uso do GT tem se mostrado uma ferramenta útil para avaliar o comportamento social em períodos de interesse, como é o caso da pandemia da COVID-19.

\section{Referências}

Angelini, M., \& Zago, V. (2021). As implicações do cenário pandêmico do COVID-19 frente a Segurança Alimentar e Nutricional: uma revisão bibliográfica. Segur. Aliment. Nutr. 61, 1-14.

Arshad Ali, S., Bin Arif, T., Maab, H., Baloch, M., Manazir, S., Jawed, F., \& Ochani, R. K. (2020). Global Interest in Telehealth During COVID-19 Pandemic: An Analysis of Google Trends ${ }^{\mathrm{TM}}$. Cureus, 12(9). 
Braga, J. C. B., \& Silva, L. R. da. (2021). Consumo de plantas medicinais e fitoterápicos no Brasil: perfil de consumidores e sua relação com a pandemia de COVID-19. Brazilian Journal of Health Review, 4(1), 3831-3839.

Brasil. Associação Brasileira de Nutrição [ASBRAN], (2020). Guia para uma alimentação saudável em tempos de COVID-19. https://www.asbran.org.br/noticias/asbran-lanca-guia-para-orientar-populacao-sobre-alimentacao-em-tempos-de-covid-19

Brasil. Ministério do Desenvolvimento Social e Combate à Fome. (2006). Lei de Segurança Alimentar e Nutricional. Lei Orgânica de Segurança Alimentar e Nutricional. Lei $N^{\circ} 11.346$ de 15 de Setembro de 2006. Cria o Sistema Nacional de Segurança Alimentar e Nutricional - SISAN Com Vistas Em Assegurar o Direito Humano à Alimentação Adequada e Dá Outras Providências, 28.

Brasil. (2004). Resolução - RDC nº 48, de 16 de março de 2004. Ministério da Saúde. Agência Nacional de Vigilância Sanitária. 1.

Carpagnano, G. E., Di Lecce, V., Quaranta, V. N., Zito, A., Buonamico, E., Capozza, E., Palumbo, A., Di Gioia, G., Valerio, V. N., \& Resta, O. (2021). Vitamin D deficiency as a predictor of poor prognosis in patients with acute respiratory failure due to COVID-19. Journal of Endocrinological Investigation, 44(4), 765-771.

Gurgel, A. D. M., Santos, C. C. S., Alves, K. P., Araujo, J. M., \& Leal, V. S. (2020). Government strategies to ensure the human right to adequate and healthy food facing the COVID-19 pandemic in Brazil. Ciencia e Saude Coletiva, 25(12), 4945-4956.

Jung, J. H., Kim, E. A., Lee, S. Y., Moon, J. E., Lee, E. J., \& Park, S. H. (2021). Vitamin d status and factors associated with vitamin d deficiency during the first year of life in preterm infants. Nutrients, 13(6), 1-11.

Kamiński, M., Skonieczna-Żydecka, K., Nowak, J. K., \& Stachowska, E. (2020). Global and local diet popularity rankings, their secular trends, and seasonal variation in Google Trends data. Nutrition, 79-80.

Lima, J. F. de. (2021). O papel da vitamina D na pandemia de COVID-19: revisão integrativa. Revista de Saúde Pública Do Paraná, 3(2), 169-178.

Manson, J. E., Brannon, P. M., Rosen, C. J., \& Taylor, C. L. (2016). Vitamin D Deficiency - Is There Really a Pandemic? The New England journal of medicine, 375(19), 1817-1820.

Mavragani, A., \& Ochoa, G. (2019). Google trends in infodemiology and infoveillance: Methodology framework. JMIR Public Health and Surveillance, 5(2), $1-15$.

Nuti, S. V., Wayda, B., Ranasinghe, I., Wang, S., Dreyer, R. P., Chen, S. I., \& Murugiah, K. (2014). The use of google trends in health care research: A systematic review. PLOS ONE, 9(10).

Organização Pan-Americana de Saúde [OPAS] (2019). Alimentação Saudável. https://www.paho.org/pt/topicos/alimentacao-saudavel

Passos, J. A., Vasconcellos-Silva, P. R., \& Santos, L. A. da S. (2020). Cycles of attention to fad diets and internet search trends by google trends. Ciencia e Saude Coletiva, 25(7), 2615-2631.

Rahimi Pordanjani, S., Hasanpour, A., Askarpour, H., Bastam, D., Rafiee, M., Khazaei, Z., Mazaheri, E., Vaziri, M. H., \& Sabour, S. (2021). Aspects of Epidemiology, Pathology, Virology, Immunology, Transmission, Prevention, Prognosis, Diagnosis, and Treatment of COVID-19 Pandemic: A Narrative Review. International journal of preventive medicine, 12, 38.

Ros, D. C., Brasioli, M., \& Guilherme, R. C. (2020). Guia para uma alimentação saudável em tempos de COVID-19. Associação Brasileira de Nutrição, 14.

Santos, M. V. A., Oliveira, I. G. de, Pereira, F. M. N., \& Santos, P. R. (2021). Insegurança alimentar e nutricional: uma análise sobre as políticas públicas de interface com alimentação e nutrição em meio a pandemia por Sars-CoV-2. Segurança Alimentar e Nutricional, 28.

Sousa, L. O. de, Rodrigues, D. B. S., Silva, R. G. da, Cardoso, A. V. S., Freitas, A. S., Cruz, B. R. dos S., Conceição, R. M. da, \& Campos, D. C. dos S. (2021). Importância Dos Alimentos No Fortalecimento Da Imunidade Frente À COVID-19. Brazilian Journal of Development, 7(3), $29842-29852$.

Valeriano, F. R., Savani, F. R., \& Silva, M. R. V. da. (2019). O uso de plantas medicinais e o interesse pelo cultivo comunitário por moradores do bairro São Francisco, município de Pitangui, MG. Interações (Campo Grande), 891-905. 